\title{
EQUATIONS FOR ABELIAN SUBVARIETIES
}

\author{
ANGEL CAROCCA, HERBERT LANGE AND RUBÍ E. RODRÍGUEZ
}

\begin{abstract}
Given a finite group $G$ and an abelian variety $A$ acted on by $G$, to any subgroup $H$ of $G$, we associate an abelian subvariety $A_{H}$ on which the associated Hecke algebra $\mathcal{H}_{H}$ for $H$ in $G$ acts. Any irreducible rational representation $\widetilde{W}$ of $\mathcal{H}_{H}$ induces an abelian subvariety of $A_{H}$ in a natural way. In this paper we give equations for this abelian subvariety. In a special case these equations become much easier. We work out some examples.
\end{abstract}

\section{INTRODUCTION}

Let $A$ be a complex abelian variety acted on by a finite group $G$. This induces an algebra homomorphism of the rational group ring $\mathbb{Q}[G]$ into the rational endomorphism $\operatorname{ring} \operatorname{End}_{\mathbb{Q}}(A)=\operatorname{End}(A) \otimes \mathbb{Q}$,

$$
\rho: \mathbb{Q}[G] \rightarrow \operatorname{End}_{\mathbb{Q}}(A) .
$$

For any element $\alpha \in \mathbb{Q}[G]$ we define its image in $A$ by

$$
\operatorname{Im}(\alpha):=\operatorname{Im}(n \alpha)
$$

where $n$ is any positive integer such that $n \alpha$ is an endomorphism. Since multiplication by a non-zero integer on $A$ is an isogeny, the definition does not depend on the chosen integer $n$.

Now for any subgroup $H$ of $G$ the element $p_{H}:=\frac{1}{|H|} \sum_{h \in H} h$ is an idempotent in $\mathbb{Q}[G]$ which defines an abelian subvariety of $A$,

$$
A_{H}:=\operatorname{Im}\left(p_{H}\right) \text {. }
$$

The action of $G$ on $A$ induces an action of the Hecke algebra $\mathcal{H}_{H}$ on the abelian subvariety $A_{H}$. The aim of this note is to study this action and use it to find equations for the abelian subvarieties $A_{H, \widetilde{W}}$ of $A_{H}$ which are given by the rational representations $\widetilde{W}$ of $\mathcal{H}_{H}$. Here "equation" means to express $A_{H}$ as the connected component containing 0 of the zeroset of an endomorphism of $A_{H}$. Since this is fairly complicated, we do not repeat the result here, but refer to Theorem 3.6 below. However, we get an easy and important consequence: If the group $G$ acts on an abelian variety $A$ and $H$ is any subgroup of $G$, then Corollary 3.3 describes the complement of the abelian subvariety $A_{G}$ in $A_{H}$. This generalizes [9, Corollary 3.5, p.10] where it is proven for the Prym varieties $P(C / H, C / G)$ for any curve $C$ acted on by $G$ and any subgroup $H$ of $G$.

Date: April 8, 2019.

1991 Mathematics Subject Classification. 14H40, 14H30.

Key words and phrases. Abelian Varieties, Jacobians, Prym Varieties.

The authors were partially supported by grants CONICYT PAI Atracción de Capital Humano Avanzado del Extranjero PAI80160004 and Anillo ACT 1415 PIA-CONICYT. 
In a special case the result becomes much simpler, namely let $V$ be an irreducible complex representation with $\operatorname{dim} V^{H}=1$ and rational character. To this a one-dimensional rational representation $\widetilde{W}$ of the Hecke algebra $\mathcal{H}_{H}$ is associated in a natural way and one can find an explicit basis $q_{1}, \ldots, q_{s}$ of the algebra $\mathcal{H}_{H}$ for which we have (see Theorem 4.3) for the isotypical component $A_{H, \widetilde{W}}$ corresponding to $\widetilde{W}$ in $A_{H}$,

Theorem 1.1. With these assumptions the abelian subvariety $A_{H, \widetilde{W}}$ is

$$
A_{H, \widetilde{W}}=\left\{z \in A_{H} \mid q_{i}(z)=\chi_{V}\left(q_{i}\right) z \text { for } 1 \leq i \leq s\right\}_{0},
$$

where the index 0 mean the connected component containing 0.

Several examples for this theorem will be given.

Section 2 contains some preliminaries. In particular we recall from [4] the decompositions of $A_{H}$ induced by the Hecke algebra $\mathcal{H}_{H}$. Section 3 contains the proof of the general theorem (Theorem 3.6). In Section 4 we prove the above mentioned theorem. Finally Section 5 contains some examples.

\section{Preliminaries}

Let $G$ be a finite group acting on an abelian variety $A$ of dimension $g$ over the field of complex numbers and let $H \leq G$ be a subgroup of $G$. The Hecke algebra for $H$ in $G$ acts in a natural way on the abelian variety $A$. To be more precise: the element

$$
p_{H}:=\frac{1}{|H|} \sum_{h \in H} h
$$

is an idempotent in the group algebra $\mathbb{Q}[G]$.

The (rational) Hecke algebra for $H$ in $G$ is defined as the subalgebra

$$
\mathcal{H}_{H}:=p_{H} \mathbb{Q}[G] p_{H}=\mathbb{Q}[H \backslash G / H]
$$

of the rational group group algebra $\mathbb{Q}[G]$. If we consider $\mathbb{Q}[G]$ as the algebra of functions $G \rightarrow \mathbb{Q}$ with multiplication the convolution product (see $[5, \S 11]$ ), then $\mathbb{Q}[H \backslash G / H]$ is the subalgebra of functions which are constant on each double coset $H g H$, and $p_{H}$ is the unit in this algebra.

The action of $G$ on $A$ induces an action of $\mathbb{Q}[G]$ on $A$ in a natural way, giving an algebra homomorphism

$$
\mathbb{Q}[G] \rightarrow \operatorname{End}_{\mathbb{Q}}(A) .
$$

Since this homomorphism is canonical, we denote the elements of $\mathbb{Q}[G]$ and their images by the same letter. For any element $\alpha \in \mathbb{Q}[G]$ we define its image in $A$ by

$$
\operatorname{Im}(\alpha):=\operatorname{Im}(n \alpha) \subset A
$$

where $n$ is any positive integer such that $n \alpha$ is in $\operatorname{End}(A)$. It is an abelian subvariety of $A$ which does not depend on the chosen integer $n$.

Consider the abelian subvariety of $A$ given by

$$
A_{H}:=\operatorname{Im}\left(p_{H}\right) .
$$


Restricting (2.1) to $\mathcal{H}_{H}$ gives an algebra homomorphism

$$
\mathcal{H}_{H} \rightarrow \operatorname{End}_{\mathbb{Q}}\left(A_{H}\right)
$$

The aim of this section is to recall from 4 the isotypical and Hecke algebra decompositions of $A_{H}$ with respect to this action of $\mathcal{H}_{H}$.

Let $\left\{W_{1}, \ldots, W_{r}\right\}$ denote the irreducible rational representations of $G$. To any $W_{i}$ there corresponds an irreducible complex representations $V_{i}$, uniquely determined up to an element of the Galois group of $K_{i}$ over $\mathbb{Q}$, where $K_{i}$ is the field obtained by adjoining to $\mathbb{Q}$ the values of the character $\chi_{V_{i}}$ of $V_{i}$. The representations $W_{i}$ and $V_{i}$ are said to be Galois associated.

To each $W_{i}$ we can associate a central idempotent $e_{W_{i}}$ of $\mathbb{Q}[G]$ by

$$
e_{W_{i}}=\frac{\operatorname{dim}_{\mathbb{C}}\left(V_{i}\right)}{|G|} \sum_{g \in G} \operatorname{tr}_{K_{i} / \mathbb{Q}}\left(\chi_{V_{i}}\left(g^{-1}\right)\right) g .
$$

Let $\rho_{H}$ denote the representation of $G$ induced by the trivial representation of $H$. It decomposes as

$$
\rho_{H} \simeq \sum_{i=1}^{r} a_{i} W_{i}
$$

with $a_{i}=\frac{1}{s_{i}} \operatorname{dim}_{\mathbb{C}}\left(V_{i}^{H}\right)$ and $s_{i}$ the Schur index of $V_{i}$. Renumbering if necessary, let $\left\{W_{i}, \ldots, W_{t}\right\}$ denote the set of all irreducible rational representations of $G$ such that $a_{i} \neq$ 0 . Then there is a bijection from this set to the set $\left\{\widetilde{W}_{1}, \ldots, \widetilde{W}_{t}\right\}$ of all irreducible rational representations of the algebra $\mathcal{H}_{H}$. An analogous statement holds for the irreducible complex representations of $G$ and of $\mathbb{C}[H \backslash G / H]$. Let $\widetilde{V}$ denote the representation of $\mathbb{C}[H \backslash G / H]$ associated to the complex irreducible representation $V$ of $G$.

According to [4, equation (2.4)] and [3, p. 331] the dimension of $\widetilde{W}_{i}$ given by

$$
\operatorname{dim}_{\mathbb{Q}}\left(\widetilde{W}_{i}\right)=\operatorname{dim}_{\mathbb{Q}}\left(W_{i}^{H}\right)=\left[L_{i}: \mathbb{Q}\right] \operatorname{dim}_{\mathbb{C}}\left(V_{i}^{H}\right) .
$$

where $L_{i}$ denotes the field of definition of the representation $V_{i}$. Recall that the index $s_{i}=\left[L_{i}: K_{i}\right]$ is the Schur index of $V_{i}$.

For $i=1, \ldots, t$ consider the central idempotents of $\mathcal{H}_{H}$,

$$
f_{H, \widetilde{W}_{i}}:=p_{H} e_{W_{i}}=e_{W_{i}} p_{H} .
$$

Then $p_{H}$ decomposes as

$$
p_{H}=\sum_{i=1}^{t} f_{H, \widetilde{W}_{i}} .
$$

Defining for $i=1, \ldots, t$ the abelian subvarieties

$$
A_{H, \widetilde{W}_{i}}:=\operatorname{Im}\left(f_{H, \widetilde{W}_{i}}\right),
$$

one obtains the following isogeny decomposition of $A_{H}$, given by the addition map

$$
+: A_{H, \widetilde{W}_{1}} \times A_{H, \widetilde{W}_{2}} \times \cdots \times A_{H, \widetilde{W}_{t}} \rightarrow A_{H} .
$$

It is uniquely determined by $H$ and the action of $G$ and called the isotypical decomposition of $A_{H}$. 
If $a_{i} \geq 2$ in (2.3), the subvarieties $A_{H, \widetilde{W}_{i}}$ can be decomposed further. In fact, given $\widetilde{W}_{i}$, explicit orthogonal primitive idempotents $f_{i, j}, 1 \leq j \leq n_{i}:=\frac{1}{s_{i}} \operatorname{dim} V_{i}$ may be found such that

$$
e_{W_{i}}=f_{i, 1}+\cdots+f_{i, n_{i}} .
$$

Multiplying by $p_{H}$ gives

$$
f_{H, \widetilde{W}_{i}}=f_{i, 1} p_{H}+\cdots+f_{i, n_{i}} p_{H} .
$$

We label the $f_{i, j} p_{H}$ in such a way that for the first $a_{i}=\frac{1}{s_{i}} \operatorname{dim}\left(V_{i}^{H}\right)$ of them, the minimal left ideals of the simple algebra $\mathbb{Q}[G] f_{H, \widetilde{W}_{i}}$

$$
J_{i, j}:=\mathbb{Q}[G] f_{i, j} p_{H} \quad \text { are different from } 0,
$$

and different among themselves; that is, such that

$$
\mathbb{Q}[G] f_{H, \widetilde{W}_{i}}=\bigoplus_{j=1}^{a_{i}} J_{i, j} .
$$

Then there exist primitive idempotents $v_{i, 1}, \ldots, v_{i, a_{i}}$ in $\mathbb{Q}[G] f_{H, \widetilde{W}_{i}}$, each $v_{i, j}$ generating the corresponding ideal $J_{i, j}$, such that

$$
f_{H, \widetilde{W}_{i}}=v_{i, 1}+\cdots+v_{i, a_{i}} .
$$

Note that by construction the $v_{i, j}$ are orthogonal idempotents, not uniquely determined, since the $f_{i, j}$ are not uniquely determined. Defining for $j=1, \ldots, a_{i}$,

$$
B_{H, \widetilde{W}_{i}, j}:=\operatorname{Im}\left(v_{i, j}\right),
$$

equation (2.6) induces the following isogeny

$$
+: B_{H, \widetilde{W}_{1}, j} \times B_{H, \widetilde{W}_{2}, j} \times \cdots \times B_{H, \widetilde{W}_{a_{i}}, j} \rightarrow A_{H, \widetilde{W}_{i}} .
$$

Here the subvarieties $B_{H, \widetilde{W}_{1}, j}, \ldots, B_{H, \widetilde{W}_{a_{i}}, j}$ are pairwise isogenous. Hence combining with the isogeny (2.5), we get the following $\mathcal{H}_{H^{-}}$equivariant isogeny

$$
B_{H, \widetilde{W}_{1}, 1}^{a_{1}} \times B_{H, \widetilde{W}_{2}, 1}^{a_{2}} \times \cdots \times B_{H, \widetilde{W}_{t}, 1}^{a_{t}} \rightarrow A_{H}
$$

called the Hecke algebra decomposition of $A_{H}$ with respect to the action of $G$ and the subgroup $H$.

\section{EQUATIONS FOR THE ABELIAN SUBVARIETIES $A_{H, \widetilde{W}_{i}}$}

In this section we describe the abelian subvarieties $A_{H, \widetilde{W}_{i}}$. The same method works for the abelian subvarieties $B_{H, \widetilde{W}_{i}, j}$, which we omit however. The method relies of the following proposition.

Let $G$ be a finite group acting on the abelian variety $A$. For any idempotent $\iota$ of $\mathbb{Q}[G]$ there are two abelian subvarieties of $A$, namely

$$
A_{\iota}:=\operatorname{Im} \iota \subset A \quad \text { and } \quad C_{\iota}:=\operatorname{Im}\left(1_{G}-\iota\right) .
$$

Proposition 3.1. (1) The addition map gives an isogeny

$$
+: A_{\iota} \times C_{\iota} \rightarrow A .
$$




$$
\text { (2) } A_{\iota}=\operatorname{Ker}\left(1_{G}-\iota\right)_{0} \quad \text { and } \quad C_{\iota}=\operatorname{Ker}(\iota)_{0} \text {. }
$$

where the index 0 means the connected component containing 0.

To be more precise, for an element $\alpha \in \operatorname{End}_{\mathbb{Q}}(A)$ we denote by $\operatorname{Ker}(\alpha)_{0}$ the connected component containing 0 of some positive multiple $n \alpha$ which is an endomorphism. This does not depend on the chosen $n$.

Given a polarization of $A$, there is an analogous result for any abelian subvariety using norm-endomorphisms (see [1, Section 5.3]). One could give also a proof of the proposition introducing polarizations, but we prefer to give a direct proof.

Proof. (1): Since $\iota+\left(1_{G}-\iota\right)=1_{G}$, the addition map $+: A_{\iota} \times C_{\iota} \rightarrow A$ is surjective. To see that is is an isogeny, it suffices to show that on the level of tangent spaces it is an isomorphism. So suppose $A=V / \Lambda$ with a $\mathbb{C}$-vector space $V$ and a lattice $\Lambda$. we may choose the basis of $V$ such that the analytic representation $\rho_{a}(\iota)$ is given by the diagonal matrix $\operatorname{diag}(1, \ldots, 1,0, \ldots, 0)$ with the number of 1 's equal to $\operatorname{dim} A_{\iota}$. Hence $\rho_{a}\left(1_{G}-\iota\right)=\operatorname{diag}(0, \ldots, 0,1, \ldots 1)$ with the number of 0 's equal to $\operatorname{dim} A_{\iota}$. This implies $\operatorname{dim} C_{\iota}=\operatorname{dim} A-\operatorname{dim} A_{\iota}$ and thus the assertion.

$(2)$ : Choose a positive integer $n$ such that $n\left(1_{G}-\iota\right) \in \operatorname{End}_{\mathbb{Q}}(A)$ and consider the exact sequence

$$
0 \rightarrow \operatorname{Ker}\left(n\left(1_{G}-\iota\right)\right) \rightarrow A \stackrel{n\left(1_{G}-\iota\right)}{\rightarrow} C_{\iota} \rightarrow 0 .
$$

Certainly $A_{\iota} \subset \operatorname{Ker}\left(n\left(1_{G}-\iota\right)\right)$. Since both have the same dimension, this implies the assertion.

Now let $H$ be a subgroup of $G$ and $\iota$ be an idempotent of the corresponding Hecke algebra $\mathcal{H}_{H}$. It induces two abelian subvarieties of the abelian variety $A_{H}$, namely

$$
A_{H, \iota}:=\operatorname{Im}(\iota) \quad \text { and } \quad P_{H, \iota}:=\operatorname{Im}\left(p_{H}-\iota\right) .
$$

Notice that $p_{H}-\iota: A_{H} \rightarrow A_{H}$ is also an idempotent of $\mathcal{H}_{H}$, since $p_{H}$ is the unit element of the algebra $\mathcal{H}_{H}$ and hence its image in $\operatorname{End}_{\mathbb{Q}}\left(A_{H}\right)$ is the identity on $A_{H}$. If we denote by $\iota$ also the corresponding idempotent of $\operatorname{End}_{\mathbb{Q}}\left(A_{H}\right)$, this implies that $p_{H}-\iota$ is an idempotent of $\operatorname{End}_{\mathbb{Q}}\left(A_{H}\right)$.

\section{Proposition 3.2. (1) The addition map gives an isogeny}

$$
\begin{gathered}
+: A_{H, \iota} \times C_{H, \iota} \rightarrow A_{H} . \\
\text { (2) } A_{H, \iota}=\operatorname{Ker}\left(p_{H}-\iota\right)_{0} \quad \text { and } \quad P_{H, \iota}=\operatorname{Ker}(\iota)_{0} .
\end{gathered}
$$

The proof is essentially the same as the proof of Proposition 3.1. We want to use the proposition to describe the isotypical components of $A_{H}$ as fixed-point sets of particular endomorphisms of $A_{H}$.

First we consider the trivial representation $W_{0}$ of $G$ and any subgroup $H$ of $G$. Note that

$$
e_{W_{0}}:=p_{G}=\frac{1}{|G|} \sum_{g \in G} g
$$

is the central idempotent in $\mathbb{Q}[G]$ corresponding to $W_{0}$. Moreover, choose a set of representatives $g_{1}, \ldots, g_{s}$ for both the right and left cosets of $H$ in $G$. Such a set exists according to [6, Theorem 5.1.7]. Using this, we get as a special case, 
Corollary 3.3. With the above notations we have for the abelian subvariety $A_{G}=\left(A^{G}\right)_{0}$ and the complement $P\left(A_{H}, A_{G}\right)$ of $A_{G}$ in $A_{H}=\operatorname{Im}\left(p_{H}\right)$,

(i) $A_{G}=\left\{z \in A_{H}: \sum_{j=1}^{s} g_{j}(z)=z\right\}_{0}$,

(ii) $P\left(A_{H}, A_{G}\right)=\left\{z \in A_{H}: \sum_{j=1}^{s} g_{j}(z)=0\right\}_{0}$.

The notation $P\left(A_{H}, A_{G}\right)$ comes from the fact that in the case of a Galois cover of curves $C \rightarrow C / G$ and any subgroup $H$ of $G$ this is just the Prym variety $P(C / H, C / G)$. In fact, Corollary 3.3 generalizes $[9$, Corollary 3.5, p.10] where it is proven for these Prym varieties $P(C / H, C / G)$.

Proof. We may assume that $H \lesseqgtr G$. Then we have

$$
p_{H}=\left(p_{H}-e_{W_{0}}\right)+e_{W_{0}} .
$$

Observe that because of the special choice of the $g_{i}$,

$$
[G: H] e_{W_{0}}=p_{H}\left(\sum_{j=1}^{s} g_{s}\right)=\left(\sum_{j=1}^{s} g_{j}\right) p_{H} .
$$

Hence $e_{W_{0}}$ is in $\mathcal{H}_{H}$ and furthermore $\operatorname{Im}\left(e_{W_{0}}\right)=A_{G}$. The assertion now follows from Proposition 3.2, since

$$
A_{G}=\operatorname{Ker}\left(p_{H}-e_{W_{0}}\right)_{0}=\left\{z \in A_{H}: \sum_{j=1}^{s} g_{j}(z)=z\right\}_{0}
$$

and

$$
P\left(A_{H}, A_{G}\right)=\operatorname{Ker}\left(e_{W_{0}}\right)_{0}=\left\{z \in A_{H}: \sum_{j=1}^{s} g_{j}(z)=0\right\}_{0}
$$

and $A_{H}=\left(A^{H}\right)_{0}$.

Let $\widetilde{W}$ be the irreducible rational representation of the Hecke algebra $\mathcal{H}_{H}$ associated to the irreducible rational representation $W$ of $G$ and

$$
f_{H, \widetilde{W}}=p_{H} e_{W}=e_{W} p_{H}
$$

the corresponding central idempotent of $\mathcal{H}_{H}$. In order to find a more convenient expression of $f_{H, \widetilde{W}}$, consider the decomposition of $G$ into double cosets of $H$ in $G$,

$$
G=H_{1} \cup H_{2} \cup \cdots \cup H_{s}
$$

with $H_{i}=H x_{i} H$ and $x_{1}=1$, i.e. $H_{1}=H$. A basis for the Hecke algebra $\mathcal{H}_{H}$ is given by the elements

$$
q_{i}:=\frac{|H|}{\left|H \cap x_{i} H x_{i}^{-1}\right|} p_{H} x_{i} p_{H}=\frac{1}{|H|} \sum_{h_{i} \in H_{i}} h_{i}
$$

for $i=1, \ldots, s$ (see [5, Proposition 11.30(i)]). For the last equation use that

$$
\frac{|H|}{\left|H \cap x_{i} H x_{i}^{-1}\right|}=\left|H: H \cap x_{i} H x_{i}^{-1}\right|=\frac{\left|H x_{i} H\right|}{|H|}=\frac{\left|H_{i}\right|}{|H|}
$$


Let $\left\{g_{i, j}, j=1, \ldots, d_{i}:=\left|H: x_{i} H x_{i}^{-1}\right|\right\}$ denote a set of simultaneous representatives for the right and left cosets for $H$ in $H_{i}$. Such a set exists again by [6, Theorem 5.1.7]. Then we have,

Lemma 3.4. Considering the elements $q_{i}$ as elements of $\operatorname{End}_{\mathbb{Q}}\left(A_{H}\right)$, we have: $q_{i}$ is an endomorphism of $A_{H}$ and as such

$$
q_{i}(z)=\sum_{j=1}^{d_{i}} g_{i, j}(z)
$$

for each $z \in A_{H}$.

Proof. Since $H_{i}=\cup_{j=1}^{d_{i}} H g_{i, j}=\cup_{j=1}^{d_{i}} g_{i, j} H$, we have,

$$
q_{i}=\frac{1}{|H|} \sum_{x \in H_{i}} x=p_{H}\left(\sum_{j=1}^{d_{i}} g_{i, j}\right)=\left(\sum_{j=1}^{d_{i}} g_{i, j}\right) p_{H} .
$$

This gives the assertion, since $p_{H}$ is the identity on $A_{H}$.

Lemma 3.5. The following equality is valid in the Hecke algebra $\mathcal{H}_{H}$ :

$$
f_{H, \widetilde{W}}=\frac{\operatorname{dim} V}{|G|} \sum_{i=1}^{s}\left(\left|H \cap x_{i} H x_{i}^{-1}\right| \operatorname{tr}_{K / \mathbb{Q}}\left(\chi_{V}\left(q_{i}\right)\right)\right) q_{i}
$$

Proof. Since $e_{W}$ is central and $p_{H}^{2}=p_{H}$, we have

$$
\begin{aligned}
f_{H, \widetilde{W}}=p_{H} e_{W} & =p_{H} e_{W} p_{H}=\frac{\operatorname{dim} V}{|G|} \sum_{g \in G} \operatorname{tr}_{K / \mathbb{Q}}\left(\chi_{V}\left(g^{-1}\right)\right)\left(p_{H} g p_{H}\right) \\
& =\frac{\operatorname{dim} V}{|G|} \sum_{i=1}^{s} \sum_{g \in H_{i}} \operatorname{tr}_{K / \mathbb{Q}}\left(\chi_{V}\left(g^{-1}\right)\right) p_{H} g p_{H} \\
& =\frac{\operatorname{dim} V}{|G|} \sum_{i=1}^{s}\left(\sum_{g \in H_{i}} \operatorname{tr}_{K / \mathbb{Q}}\left(\chi_{V}\left(g^{-1}\right)\right) p_{H} x_{i} p_{H}\right) \\
& =\frac{\operatorname{dim} V}{|G|} \sum_{i=1}^{s}\left(\sum_{g \in H_{i}} \frac{\left|H \cap x_{i} H x_{i}^{-1}\right|}{|H|} \operatorname{tr}_{K / \mathbb{Q}}\left(\chi_{V}\left(g^{-1}\right)\right)\right) \frac{|H|}{\left|H \cap x_{i} H x_{i}^{-1}\right|} p_{H} x_{i} p_{H} .
\end{aligned}
$$

But for any $g \in G$ we have $\chi_{V}\left(g^{-1}\right)=\overline{\chi_{V}(g)}$, and therefore

$$
\sum_{g \in H_{i}} \operatorname{tr}_{K / \mathbb{Q}}\left(\chi_{V}\left(g^{-1}\right)\right)=\sum_{g \in H_{i}} \operatorname{tr}_{K / \mathbb{Q}}\left(\chi_{V}(g)\right)=|H| \operatorname{tr}_{K / \mathbb{Q}}\left(\chi_{V}\left(q_{i}\right)\right) .
$$

So equation (3.2) gives the assertion.

Recall that we consider any element of $\mathbb{Q}[G]$ also as en element of $\operatorname{End}_{\mathbb{Q}}(A)$. We denote for any element $\alpha$ of $\mathcal{H}_{H} \subset \mathbb{Q}[G]$ by

$$
\operatorname{Ker}(\alpha)_{0}
$$


the connected component of $\operatorname{Ker}(n \alpha) \subset A_{H}$ containing 0 , where $n$ is any positive integer such that $n \alpha$ is actually an endomorphism. This does not depend of the chosen $n$. Then we get the following equation for the abelian subvariety $A_{H, \widetilde{W}}$ of $A_{H}$.

Theorem 3.6. The isotypical component $A_{H, \widetilde{W}}$ of $A_{H}$ is given by

$$
A_{H, \widetilde{W}}=\operatorname{Ker}\left(p_{H}-\frac{\operatorname{dim} V}{|G|} \sum_{i=1}^{s}\left(\left|H \cap x_{i} H x_{i}^{-1}\right| \operatorname{tr}_{K / \mathbb{Q}}\left(\chi_{V}\left(q_{i}\right)\right)\right) q_{i}\right)_{0} .
$$

Proof. By definition of $A_{H, \widetilde{W}}$ and Proposition 3.2 we have

$$
A_{H, \widetilde{W}}=\operatorname{Im}\left(f_{H, \widetilde{W}}\right)=\operatorname{Ker}\left(p_{H}-f_{H, \widetilde{W}}\right)_{0} .
$$

So Lemma 3.5 gives the assertion.

In the next section we will use the following orthogonality relations,

Lemma 3.7. Let $\widetilde{U}, \widetilde{V}$ be complex irreducible representations of $\mathbb{C}[H \backslash G / H]$ associated to the irreducible representations $U, V$ of $G$. Then, with the notation of above,

$$
\sum_{j=1}^{s} \frac{|H|}{\left|H \cap x_{j} H x_{j}^{-1}\right|} \chi_{\widetilde{U}}\left(q_{j}^{-1}\right) \chi_{\widetilde{V}}\left(q_{j}\right)=\left\{\begin{array}{lll}
0 & \text { if } & \widetilde{U} \neq \widetilde{V} \\
\frac{[G: H]}{\operatorname{dim} U} & \text { if } & \widetilde{U}=\widetilde{V}
\end{array}\right.
$$

where $q_{j}^{-1}:=\frac{1}{|H|} \sum_{y \in H_{j}} y^{-1}$.

Proof. The orthogonality relations [5, Theorem 11.32 (ii)] say, in the special case that $\psi$ is the trivial representation,

$$
\sum_{j=1}^{s} \frac{1}{\left[H: H \cap x_{j} H x_{j}^{-1}\right]} \chi_{\widetilde{U}}\left(\frac{1}{|H|} \sum_{y \in H_{j}} y^{-1}\right) \chi_{\widetilde{V}}\left(\frac{1}{|H|} \sum_{x \in H_{j}} x\right)= \begin{cases}0, & \text { if } \tilde{U} \neq \widetilde{V} \\ \frac{[G: H]}{\operatorname{dim} V} & \text { if } \quad \widetilde{U}=\widetilde{V}\end{cases}
$$

This implies the assertion.

The following lemma is proven in [5, p.282, 1. -4].

Lemma 3.8. Let $\widetilde{V}$ be a complex irreducible representation of $\mathbb{C}[H \backslash G / H]$ associated to the irreducible representation $V$ of $G$. Then their characters satisfy

$$
\chi_{\widetilde{V}}(x)=\chi_{V}(x) \quad \text { for all } \quad x \in \mathbb{C}[H \backslash G / H] .
$$

\section{A sPecial CASE}

The equation of Theorem 3.6 seems fairly complicated. In a special case we can describe the subvariety $A_{H, \widetilde{W}}$ in a simpler way. Let the notation be as above, but assume in addition that

$$
\operatorname{dim} V^{H}=1 \quad \text { and } \quad K=\mathbb{Q} .
$$

According to [7, Corollary 10.2], this implies that also the Schur index $s(V)$ of $V$ is equal to 1 . Then we have, according to equation (2.4),

$$
\operatorname{dim} \widetilde{W}=\operatorname{dim} W^{H}=1
$$


This implies that the complex representation $\widetilde{V}$ of $\mathbb{C}[H \backslash G / H]$ is rational of dimension 1 with $\widetilde{W}=\widetilde{V} \otimes \mathbb{C}$, and the complex representation $V$ of $G$ is rational with $W=V \otimes \mathbb{C}$.

Proposition 4.1. With the assumption (4.1) we have

$$
f_{H, \widetilde{W}}=\frac{\operatorname{dim} V}{|G|} \sum_{i=1}^{s}\left|H \cap x_{i} H x_{i}^{-1}\right| \chi_{V}\left(q_{i}\right) q_{i} .
$$

Proof. This is a direct consequence of Lemma 3.5.

Now consider all elements of $\mathcal{H}_{H}$ as elements of $\operatorname{End}_{\mathbb{Q}}\left(A_{H}\right)$. According to Lemma 3.4, $q_{i}$ is an endomorphism of $A_{H}$ for all $i$ and we can express $A_{H, \widetilde{W}}$ as the kernel of an actual endomorphism. In fact, we get as a direct consequence of Proposition 4.1 and Theorem 3.6.

Corollary 4.2. With the assumption (4.1) let $n:=|G|$. Then $n f_{H, \widetilde{W}}$ is an endomorphism of $A_{H}$ and we have

$$
A_{H, \widetilde{W}}=\operatorname{Ker}\left(n_{A_{H}}-\operatorname{dim} V \sum_{i=1}^{s}\left|H \cap x_{i} H x_{i}^{-1}\right| \chi_{V}\left(q_{i}\right) q_{i}\right)_{0} .
$$

Define the abelian subvariety $B_{H, \widetilde{W}}$ by

$$
B_{H, \widetilde{W}}:=\left\{z \in A_{H} \mid q_{i}(z)=\chi_{V}\left(q_{i}\right) z \text { for } i=2, \ldots, s\right\}_{0} .
$$

Note that the condition $q_{1}(z)=\chi_{V}\left(q_{1}\right) z(=z)$ is hidden the the assumption $z \in A_{H}$. So one could equivalently write

$$
B_{H, \widetilde{W}}=\left\{z \in A \mid q_{i}(z)=\chi_{V}\left(q_{i}\right) z \text { for } i=1, \ldots, s\right\}_{0} .
$$

The aim of this section is the proof of the following theorem.

Theorem 4.3. Under the assumptions (4.1) we have

$$
A_{H, \widetilde{W}}=B_{H, \widetilde{W}} .
$$

Recall that $\left\{f_{H, \widetilde{W}}\right\}_{\widetilde{W}}$ are the central primitive idempotents in $\mathcal{H}_{H}$ and the isotypical decomposition of $\mathcal{H}_{H}$ is

$$
\mathcal{H}_{H}=\oplus_{\widetilde{W}} \mathcal{H}_{H} f_{H, \widetilde{W}}
$$

where $\widetilde{W}$ acts on the simple subalgebra $\mathcal{H}_{H} f_{H, \widetilde{W}}$ and by 0 on the other components. Recall moreover that the $q_{i}, i=1, \ldots, s$ as defined in (3.2) are a basis of $\mathcal{H}_{H}$.

Lemma 4.4. Under the assumption (4.1) the action of the Hecke algebra $\mathcal{H}_{H}$ on the abelian variety $A_{H, \widetilde{W}}$ is given by

$$
q_{i}(z)=\chi_{V}\left(q_{i}\right) z \quad \text { for all } z \in A_{H, \widetilde{W}} \text { and for all } i=1, \ldots, s .
$$

Proof. Note first that the left hand side of the equation makes sense, since $q_{i}$ is an endomorphism on $A_{H}$ by Lemma 3.4. In order to see that also the right hand side makes sense, we have to show that $\chi_{V}\left(q_{i}\right)$ is an integer. But [8, Lemma 7.1] says that for any subgroup $H$ of any finite group $G$ and any complex representation $V$ of $G$ the numbers $\chi_{V}\left(q_{i}\right)$ are algebraic integers. Since in our case $\chi_{V}$ has rational values, this means $\chi_{V}\left(q_{i}\right)$ is an integer. 
For the proof of the lemma, it suffices to show that the analogous equation is valid for the action of $\mathcal{H}_{H}$ on $\mathcal{H}_{H} f_{H, \widetilde{W}}$ by $\widetilde{W}$, i.e. to show

$$
q_{i}(x)=\chi_{V}\left(q_{i}\right) x \quad \text { for all } x \in \mathcal{H}_{H} f_{H, \widetilde{W}} .
$$

Since $\widetilde{W}$ is of dimension one, we have $\widetilde{V}=\widetilde{W} \otimes \mathbb{C}$ and $\mathcal{H}_{H} f_{H, \widetilde{W}}$ is a simple algebra of dimension 1 , hence equal to $\mathcal{H}_{H} f_{H, \widetilde{W}}=\mathbb{Q} f_{H, \widetilde{W}}$. But the action of a one-dimensional complex representation is given by multiplication by the character (which equals the representation). This implies in particular $q_{i}(x)=\chi_{\widetilde{V}}\left(q_{i}\right) x$ for all $\mathrm{i}$ and all $x \in \mathcal{H}_{H} f_{H, \widetilde{W}}$. Since $\chi_{\widetilde{V}}=\chi_{V}$ on $\mathcal{H}_{H}$ by Lemma 3.8, this gives the assertion. This completes the proof of the theorem.

Proof of Theorem 4.3. Recall that

$$
A_{H, \widetilde{W}}=\operatorname{Im}\left(|G| f_{H, \widetilde{W}}\right)=\left\{z \in A_{H}|| G\left|f_{H, \widetilde{W}}(z)=\right| G \mid z\right\}_{0}
$$

First we show $A_{H, \widetilde{W}} \subset B_{H, \widetilde{W}}$ : Suppose that $z \in A_{H, \widetilde{W}}$. Since $q_{i}=q_{i} f_{H, \widetilde{W}}$, we get for ail $i$,

$$
\begin{aligned}
|G| q_{i}(z) & =|G| q_{i} f_{H, \widetilde{W}}(z) \\
& =\chi_{V}\left(q_{i}\right)|G| f_{H, \widetilde{W}}(z) \quad \text { (by Lemma 4.4) } \\
& =\chi_{V}\left(q_{i}\right)|G|(z) .
\end{aligned}
$$

So

$$
z \in\left\{z \in A_{H}|| G\left|q_{i}(z)=\chi_{V}\left(q_{i}\right)\right| G \mid z\right\}_{0}=\left\{z \in A_{H} \mid q_{i}(z)=\chi_{V}\left(q_{i}\right) z\right\}_{0}
$$

i.e. $z \in B_{H, \widetilde{W}}$.

Finally we show $B_{H, \widetilde{W}} \subset A_{H, \widetilde{W}}$ : So suppose $z \in B_{H, \widetilde{W}}$, i.e.

$$
q_{i}(z)=\chi_{V}\left(q_{i}\right) z \text { for all } i=1, \ldots, s .
$$

Since in our case $\chi_{V}\left(g^{-1}\right)=\chi_{V}(g)$ for all $g$ and $\chi_{\widetilde{V}}=\left.\chi_{V}\right|_{\mathcal{H}_{H}}$ by Lemma 3.8, part of the orthogonality relations 3.7 become

$$
\operatorname{dim} V \sum_{i=1}^{s}\left|H \cap x_{i} H x_{i}^{-1}\right| \chi_{V}\left(q_{i}\right) \chi_{V}\left(q_{i}\right)=|G| .
$$

From this we get

$$
\begin{aligned}
\operatorname{dim} V \sum_{i=1}^{s}\left|H \cap x_{i} H x_{i}^{-1}\right| \chi_{V}\left(q_{i}\right) q_{i}(z) & =\operatorname{dim} V \sum_{i=1}^{s}\left|H \cap x_{i} H x_{i}^{-1}\right| \chi_{V}\left(q_{i}\right) \chi_{V}\left(q_{i}\right)(z) \\
& =|G| z
\end{aligned}
$$

This means that $z \in A_{H, \widetilde{W}}$.

\section{EXAMPLES}

In this section we work out the equations of Theorem 4.3 in some cases. For the computations we used the computer program GAP. 
5.1. Example 1. Let $G=S_{4}$ and $W=V$ the standard representation of degree 3:

$$
\chi_{W}(1)=3, \chi_{W}(. .)=1, \chi_{W}((. .)(. .))=-1, \chi_{W}(. .)=0, \chi_{W}(\ldots .)=-1
$$

where $n$ dots mean any cycle of length $n$.

Case 1: $H=\langle(12),(34)\rangle \simeq \mathbb{Z}_{2} \times \mathbb{Z}_{2}$ (not normal in $\left.G\right)$.

Clearly the assumptions (4.1) are satisfied and

$$
G=H \cup(H(23) H) \cup(H(13)(24) H)=: H_{1} \cup H_{2} \cup H_{3} .
$$

One checks

$$
q_{1}=\frac{1}{4} \sum_{h \in H_{1}} h=p_{H}, \quad q_{2}=\frac{1}{4} \sum_{k \in H_{2}} k, \quad q_{3}=\frac{1}{4} \sum_{k \in H_{3}} k
$$

and

$$
\chi_{W}\left(q_{1}\right)=1, \quad \chi_{W}\left(q_{2}\right)=0, \quad \chi_{W}\left(q_{3}\right)=-1 .
$$

We get from Theorem 4.3 for any abelian variety $A$ with $G$-action,

$$
A_{H, \widetilde{W}}=\left\{z \in A_{H}: q_{2}(z)=0, q_{3}(z)=-z\right\}_{0} .
$$

Now observe that,

$$
H_{3}=\{(13)(24),(14)(23),(1324),(1423)\}=D_{4}-H
$$

where $D_{4}$ denotes the dihedral subgroup of $S_{4}$. This implies

$$
A_{\mathbb{Z}_{2} \times \mathbb{Z}_{2}, \widetilde{W}}=P\left(A_{\mathbb{Z}_{2} \times \mathbb{Z}_{2}} / A_{D_{4}}\right)
$$

where $P\left(A_{\mathbb{Z}_{2} \times \mathbb{Z}_{2}} / A_{D_{4}}\right)$ denotes the complement of $A_{D_{4}}$ in $A_{\mathbb{Z}_{2} \times \mathbb{Z}_{2}}$.

Case 2: $H=\langle(34),(243)\rangle \simeq S_{3}$ :

Again the assumptions (4.1) are satisfied. Here we have

$$
G=H \cup(H(14) H)=: H_{1} \cup H_{2} .
$$

One checks

$$
q_{1}=\frac{1}{6} \sum_{h \in H} h=p_{H}, \quad q_{2}=\frac{1}{6} \sum_{k \in H_{2}} k
$$

and

$$
\chi_{W}\left(q_{1}\right)=1, \quad \chi_{W}\left(q_{2}\right)=-1 .
$$

We get from Theorem 4.3 for any abelian variety $A$ with $G$-action,

$$
A_{H, \widetilde{W}}=\left\{z \in A_{H}: q_{2}(z)=-z\right\}_{0} .
$$

Now observe that $H_{2}=S_{4}-H$, which implies, as in Case 1,

$$
A_{H, \widetilde{W}}=P\left(A_{S_{3}} / A_{S_{4}}\right) .
$$


5.2. Example 2. Let $G=N \rtimes P \simeq \mathbb{Z}_{2}^{4} \rtimes \mathbb{Z}_{5}$ denote the subgroup of order 80 of $S_{10}$ (which occurred in [2]) that is generated by the permutations $s_{i}:=(i i+1)(5+i 6+i)$ for $i=1, \ldots, 4$ and $\sigma:=(12345)(678910)$.

For $i=1,2,3$ consider the rational irreducible representation $W_{i}$ defined as follows: The group $P$ acts on the character group $\widehat{N}$ of $N$ in the usual way. Apart from the trivial representation $\chi_{0}$ there are 3 orbits of the action of $P$ on $\widetilde{N}$. Let $\chi_{i}, i=1,2,3$ be representatives of them. Then, if $\rho$ denotes the representation of $G$ induced by the trivial representation of $N$, the rational irreducible representation $W_{i}$ of degree five is defined as

$$
W_{i}=\rho \otimes \chi_{i} \quad \text { for } \quad i=1,2,3 .
$$

The characters of $W_{i}$ for $i=1,2,3$ are given by $\chi_{W_{i}}\left(1_{G}\right)=5, \chi_{W_{i}}(\sigma)=0$, and

$$
\begin{aligned}
& \chi_{W_{1}}\left(s_{j}\right)=1, \quad \chi_{W_{1}}\left(s_{1} s_{2}\right)=1, \quad \chi_{W_{1}}\left(s_{1} s_{3}\right)=-3, \\
& \chi_{W_{2}}\left(s_{j}\right)=-3, \quad \chi_{W_{2}}\left(s_{1} s_{2}\right)=1, \quad \chi_{W_{2}}\left(s_{1} s_{3}\right)=1, \\
& \chi_{W_{3}}\left(s_{j}\right)=1, \quad \chi_{W_{3}}\left(s_{1} s_{2}\right)=-3, \quad \chi_{W_{3}}\left(s_{1} s_{3}\right)=1 .
\end{aligned}
$$

Case 1: $H=P$.

For the representation $\rho_{H}$ of $G$ induced by the trivial representation of $H$ we have $\rho_{H}=$ $\chi_{0} \oplus W_{1} \oplus W_{2} \oplus W_{3}$ which implies for $i=1,2,3$,

$$
\operatorname{dim} \widetilde{W}_{i}=\operatorname{dim} W_{i}^{H}=\left\langle W_{i}, \rho_{H}\right\rangle=1 .
$$

Hence the assumptions (4.1) are satisfied for all $W_{i}$. The double coset decomposition of $G$ is

$$
G=H \cup\left(H s_{1} H\right) \cup\left(H s_{1} s_{2} H\right) \cup\left(H s_{1} s_{3} H\right)=: H_{1} \cup H_{2} \cup H_{3} \cup H_{4} .
$$

The double cosets $H_{2}, H_{3}, H_{4}$ contain each a complete conjugacy class of involutions of $G$ and all other elements are of order 5. A basis of the (commutative) Hecke algebra $\mathcal{H}_{H}$ is given by

$$
q_{1}=p_{H}, \quad \text { and } \quad q_{i}=\frac{1}{5} \sum_{k_{i} \in H_{i}} k_{i} \quad \text { for } \quad i=2,3,4 .
$$

The multiplication of $\mathcal{H}_{H}$ is given by

$$
\begin{gathered}
q_{1} q_{j}=q_{j}, \quad q_{2}^{2}=5 q_{1}+2 q_{3}+2 q_{4}, \quad q_{3}^{2}=5 q_{1}+2 q_{2}+2 q_{4}, \quad q_{4}^{2}=5 q_{1}+2 q_{2}+2 q_{3}, \\
q_{2} q_{3}=2 q_{2}+2 q_{3}+q_{4}, \quad q_{2} q_{4}=2 q_{2}+q_{3}+2 q_{4}, \quad q_{3} q_{4}=q_{2}+2 q_{3}+2 q_{4} .
\end{gathered}
$$

Since

$$
\chi_{W_{i}}\left(q_{1}\right)=\chi_{W_{1}}\left(q_{2}\right)=\chi_{W_{1}}\left(q_{3}\right)=\chi_{W_{2}}\left(q_{3}\right)=\chi_{W_{2}}\left(q_{4}\right)=\chi_{W_{3}}\left(q_{2}\right)=\chi_{W_{3}}\left(q_{4}\right)=1,
$$

and

$$
\chi_{W_{1}}\left(q_{4}\right)=\chi_{W_{2}}\left(q_{2}\right)=\chi_{W_{3}}\left(q_{3}\right)=-3,
$$

we get from Theorem 4.3 that for any abelian variety $A$ with an action of the group $G$,

$$
\begin{aligned}
& A_{H, \widetilde{W}_{1}}=\left\{z \in A_{H} \mid q_{2}(z)=q_{3}(z)=z, q_{4}(z)=-3 z\right\}_{0}, \\
& A_{H, \widetilde{W}_{2}}=\left\{z \in A_{H} \mid q_{3}(z)=q_{4}(z)=z, q_{2}(z)=-3 z\right\}_{0}, \\
& A_{H, \widetilde{W}_{3}}=\left\{z \in A_{H} \mid q_{2}(z)=q_{4}(z)=z, q_{3}(z)=-3 z\right\}_{0} .
\end{aligned}
$$

Case 2: $H=\left\langle s_{1}, s_{2}, s_{3}\right\rangle \simeq \mathbb{Z}_{2}^{3}$. 
For the representation $\rho_{H}$ of $G$ induced by the trivial representation of $H$ we have

$$
\rho_{H}=\chi_{0} \oplus W_{3} \oplus \psi
$$

with $\psi=\rho_{1} \oplus \ldots \oplus \rho_{4}$ is the rational irreducible representation of degree four given by the sum of the linear complex irreducible characters of $G$ induced by each of the non-trivial characters of $P \simeq \mathbb{Z}_{5}$ by the projection $G \rightarrow P$. Hence the assumptions (4.1) are satisfied for the representation $W_{3}$.

The double coset decomposition of $G$ is

$$
\left.G=H \cup\left(H s_{4} H\right) \cup\left(H \sigma^{4} H\right) \cup\left(H \sigma^{3} H\right)\right) \cup\left(H \sigma^{2} H\right) \cup(H \sigma H)=: H_{1} \cup \cdots \cup H_{6} .
$$

where the first double coset is $H$, the second is $N-H$, and the other four consist of 16 elements of order five each.

A basis for the (commutative) Hecke algebra $\mathcal{H}_{H}$ is given by

$$
q_{1}=p_{H} \text { and } q_{j}=\frac{1}{8} \sum_{k_{j} \in H_{j}} k_{j} \text { for } j=2, \ldots, 6
$$

and one checks

$$
\begin{gathered}
q_{1} q_{j}=q_{j} \text { for all } j, \quad q_{2} q_{j}=8 q_{j} \text { for } 3 \leq l \leq 6, \\
q_{2}^{2}=64 q_{1}, \quad q_{3}^{2}=8 q_{3}, \quad q_{4}^{2}=8 q_{6}, \quad q_{5}^{2}=8 q_{3}, \quad q_{6}^{2}=8 q_{5} .
\end{gathered}
$$

Since

$$
\chi_{W_{3}}\left(q_{1}\right)=1, \chi_{W_{3}}\left(q_{2}\right)=-1 \text { and } \chi_{W_{3}}\left(q_{i}\right)=0 \text { for } i=3, \ldots, 6,
$$

we get from Theorem 4.3 ,

$$
A_{H, \widetilde{W}_{3}}=\left\{z \in A_{H} \mid q_{2}(z)=-z, q_{i}(z)=0 \text { for } i=3, \ldots, 6\right\}_{0}=P\left(A_{H} / A_{N}\right) .
$$

An analogous result can be proved for the representations $\widetilde{W}_{1}$ and $\widetilde{W}_{2}$.

\section{REFERENCES}

[1] Ch. Birkenhake, H. Lange, Complex Abelian Varieties. 2nd edition, Grundl. Math. Wiss. vol 302, Springer (2004).

[2] A. Carocca, H. Lange, R. E. Rodríguez: Étale double covers of cyclic p-gonal curves, Preprint (2018).

[3] A. Carocca, R. E. Rodríguez, Jacobians with group actions and rational idempotents. Journ. of Alg. 306 (2006), 322-343.

[4] A. Carocca, R. E. Rodríguez, Hecke algebras acting on abelian varieties. J. Pure Appl. Algebra 222 (2018), 2626-2647.

[5] C.W. Curtis, I. Reiner, Methods of representation theory, Vol. 1. John Wiley, (1981).

[6] M. Hall, Combinatorial Theory. 2nd ed., John Wiley, (1986).

[7] I. M. Isaacs, Character theory of finite groups. Academic Press, (1976).

[8] I. M. Isaacs, G. Navarro: Character sums and double cosets. Journ. of Alg. 320 (2008), 37493764 .

[9] S. Recillas, R. E. Rodríguez: Prym varieties and fourfold covers. arXiv:math/0303155.

Departamento de Matemática y Estadística, Universidad de la Frontera, Avenida Francisco Salazar 01145, Casilla 54-D, Temuco, Chile.

E-mail address: angel.carocca@ufrontera.cl 
Department Mathematik, Universität Erlangen, Cauerstrasse 11, 91058 Erlangen, GERMANY.

E-mail address: lange@math.fau.de

Departamento de Matemática y Estadística, Universidad de la Frontera, Avenida Francisco Salazar 01145, Casilla 54-D, Temuco, Chile.

E-mail address: rubi.rodriguez@ufrontera.cl 